\title{
Surgical Education
}

Anip Joshi 1

\begin{abstract}
${ }^{1}$ Assistant Professor of Surgery and Member of Subject Committee of MS General Surgery Program, Bir Hospital, National Academy of Medical Sciences, Kathmandu, Nepal
\end{abstract}

\section{Dear Editor,}

The paper by Shrestha $B M^{1}$ has outlined the format and objectives of academic foundation viva. I congratulate Dr. Shrestha for his paper on surgical education and I express my gratitude for sharing his experience of surgical training curriculum in the UK . I highly appreciate his work which gives information about the current curriculum of general surgery examination in the UK.

The author has emphasized on the need for inclusion of "critical appraisal of published literature as a mandatory component of specialty general surgical examinations." In our general surgery training, the surgical trainees are provided with a Research Methodology Training at the beginning of the training. The surgical trainees are encouraged to present published scientific research in the" Journal clubs" as a part of their monthly academic program. This helps the trainee to acquire skills on literature search and gives them up to date knowledge about the recent advances in surgery. Besides, the trainee critically analyses whether the study background forms the adequate scientific basis for conducting the research, whether the methodology is scientifically sound and whether the result can be introduced to change the surgical practice. Furthermore, the surgical trainee is expected to complete a thesis work within three years training under the academic guidance of a preceptor. The thesis work provides the surgical trainee with ample opportunity to gain further understanding about research proposal, research methodology, data analysis and preparation of thesis report. During the exit examination, the examiner also evaluates the trainee based on their thesis work and the trainee should be able to justify his thesis. In this setting, the need for formal assessment by critical appraisal of a scientific paper at the exit examination becomes debatable.
The author has highlighted that the "....data analysis should be done on an intention to treat (ITT) basis". As compared to ITT, The Per Protocol (PP) analysis gives the true efficacy of an intervention because it compares the treatment groups that include only the participants who completed the treatment as allocated. Although, PP analysis is not representative of a real life situation, it is recommended by the CONSORT ${ }^{2}$ guidelines to "instigate adherence to the protocol before publication". If the data analysis is done and reported both with ITT and PP analysis for all planned outcomes, it allows readers to interpret the effect of an intervention.

\section{REFERENCES}

1. Shrestha BM. Critical appraisal of published literaturein Speciality General Surgical Examinations in the United Kingdom.J Nepal Health Res Counc. 2017 ;15 (37):2901. [JNHRC]

2. Schulz KF, Altman DG, Moher D, CONSORT Group. CONSORT 2010 Statement: updated guidelines for reporting parallel group randomised trials. BMJ. 2010;340:c332.[PMC] 